\title{
Regulation of gene expression by transcription factors and RNA-binding proteins
}

\section{Udo Heinemann}

\author{
Max Delbrück Center for Molecular Medicine, Berlin, Germany \\ heinemann@mdc-berlin.de
}

In each human cell, only a subset of the genes is transcribed into RNA and translated into proteins, because gene expression is tightly regulated. The regulation of gene expression is a complex process involving several hierarchical stages, beginning in the nucleus with the control of transcription initiation by transcription factors. After maturation, mRNAs are translocated into the cytosol, where their stability, translation and, ultimately, degradation is under the control of RNA-binding proteins. The central event at all stages of gene expression regulation involves the recognition of DNA or RNA sequence motifs by transcription factors or RNA-binding proteins. Structural studies in my laboratory have highlighted crucial aspects of transcriptional and translational regulation. Transcriptional target gene recognition in bacteria (1) and eukaryotes $(2,3)$ proceeds according to common principles, but differs in crucial aspects. Half-life and translation of mRNAs are controlled by proteins often binding to the 3'-untranslated regions of mRNAs. RNA-binding proteins studied in my laboratory promote mRNA degradation by recruiting nucleolytic degradation complexes $(4,5)$ or through their intrinsic ribonuclease activity (6).

1. Khare, D. et al. (2004) Sequence-specific DNA binding determined by contacts outside the helix-turn-helix motif of the ParB homolog KorB. Nat. Struct. Mol. Biol. 11, 656-663

2. Schuetz, A. et al. (2011) The structure of the Klf4 DNA-binding domain links to self-renewal and macrophage differentiation. Cell. Mol. Life Sci. 68, 3121-3131

3. Ming, Q. et al. (2018) Structural basis of gene regulation by the Grainyhead/CP2 transcription factor family. Nucleic Acids Res. 46, 20822095

4. Mayr, F. et al. (2012) The Lin28 cold-shock domain remodels pre-let-7 microRNA. Nucleic Acids Res. 40, $7492-7506$

5. Schuetz, A. et al. (2014) Roquin binding to target mRNAs involves a winged helix-turn-helix motif. Nat. Commun. 5:5701

6. Garg, A. et al. (2021) PIN and CCCH Zn-finger domains coordinate RNA targeting in ZC3H1 family endoribonucleases. Nucleic Acids Res. 49, 5369-5381

Keywords: RNA-binding protein, immune homeostasis, protein-RNA interaction, crystal structure 